\title{
PAPER \\ Noncoherent Maximum Likelihood Detection for Differential Spatial Multiplexing MIMO Systems
}

\author{
Ziyan JIA $^{\dagger \text { a) }}$, Student Member, Katsunobu YOSHII ${ }^{\dagger}$, Nonmember, Shiro HANDA ${ }^{\dagger}$, Fumihito SASAMORI ${ }^{\dagger}$, \\ and Shinjiro OSHITA ${ }^{\dagger}$, Members
}

\begin{abstract}
SUMMARY In this paper, we propose a novel noncoherent maximum likelihood detection (NMLD) method for differential spatial multiplexing (SM) multiple-input multiple-output (MIMO) systems. Unlike the conventional maximum likelihood detection (MLD) method which needs the knowledge of channel state information (CSI) at the receiver, NMLD method has no need of CSI at either the transmitter or receiver. After repartitioning the observation block of multiple-symbol differential detection (MSDD) and following a decision feedback process, the decision metric of NMLD is derived by reforming that of MSDD. Since the maximum Doppler frequency and noise power are included in the derived decision metric, estimations of both maximum Doppler frequency and noise power are needed at the receiver for NMLD. A fast calculation algorithm (FCA) is applied to reduce the computational complexity of NMLD. The feasibility of the proposed NMLD is demonstrated by computer simulations in both slow and fast fading environments. Simulation results show that the proposed NMLD has good bit error rate (BER) performance, approaching that of the conventional coherent MLD with the extension of reference symbols interval. It is also proved that the BER performance is not sensitive to the estimation errors in maximum Doppler frequency and noise power.

key words: noncoherent maximum likelihood detection, spatial multiplexing, MIMO, multiple-symbol differential detection, fast calculation algorithm
\end{abstract}

\section{Introduction}

Multiple-input multiple-output (MIMO) [1] is an attractive and promising technique for wireless communication systems. By using multiple antennas at both the transmitter and receiver, MIMO systems promise higher data rates as well as improved performance compared to single antenna systems. There are mainly two kinds of MIMO systems: the space-time coding (STC) [2], [3] MIMO systems which are designed to increase the transmit diversity order, and the spatial multiplexing (SM) [4], [5] MIMO systems which are designed to increase the transmission data rates.

For SM MIMO systems, main detection schemes include zero-forcing (ZF), minimum mean square error (MMSE), maximum likelihood detection (MLD) [6] and Vertical Bell Laboratories Layered Space Time (V-BLAST) [7]; and all of these schemes need to know the channel state information (CSI) at the receiver. But in some situations such as very fast fading channel environment, reliable channel estimation is difficult to realize if not impossible. In such cases, noncoherent detection with differential coding, which

Manuscript received April 21, 2009.

Manuscript revised October 14, 2009.

${ }^{\dagger}$ The authors are with the Faculty of Engineering, Shinshu University, Nagano-shi, 380-8553 Japan.

a)E-mail: jiaziyan@oshan.shinshu-u.ac.jp

DOI: 10.1587/transcom.E93.B.361 circumvents the need for channel estimation, becomes more competitive.

Some noncoherent detection schemes [8]-[10] have been proposed for SM MIMO systems. In [8], a differential modulation scheme is proposed for the modified V-BLAST system [11] where delay offsets are intentionally applied to each sub-stream and zeros are inserted in the space-time code matrix to avoid interblock interference. In differential modified V-BLAST scheme (DV-BLAST), differential encoding is individually applied to each elements in the code matrix, and after source separation by passing the receive signals through matched filter banks, differential decoding can also be applied to individual elements. An assumption, that the channel is unchanged for the duration of two consecutive code matrices, is necessary for this scheme. In a fast fading environment, such an assumption becomes hard to maintain. That means the scheme is unsuited to fast fading conditions.

In [9], a differential SM (DSM) method is presented. Multiple transmit antennas are divided into groups with one or several antennas. Differential space-time or phase shift keying (PSK) encoding and noncoherent decoding are applied individually to each group. Differential maximum signal-to-noise ratio (MSNR) criterion, which is developed from the coherent MSNR [12], is used for the differential group interference suppression (GIS); and following GIS, decision feedback differential detection (DFDD) [13] is adopted for noncoherent detection. Since the quality of the estimates of correlation matrices needed for differential GIS deteriorates very fast with the increase of the time variation of the channel, it seems that DSM is also unsuited to fast fading conditions.

In [10], based on the maximum-likelihood sequence estimation (MLSE) [14] with per-survivor processing (PSP) [15], MIMO communication systems employing per transmit antenna differential encoding (PADE), has been proposed. The branch metric of PADE can be generated by a fixed finite impulse response filter with current and several previous received signals as inputs. For SM MIMO systems, though the performance of PADE can be improved by schemes proposed in [16] in slow fading environments, PADE still suffers from bit error rate (BER) floor in fast fading environments.

In this paper, we propose a novel noncoherent MLD (NMLD) method for differential SM MIMO systems which has a good performance and no BER floor in both slow and 
fast fading environments. The proposed NMLD is mainly inspired by the idea of multiple-symbol differential detection (MSDD) [17], [18]. The basic idea of MSDD is to make a joint MLD of a block of information symbols based on a corresponding block of received signals simultaneously instead of symbol-by-symbol detection. MSDD can not be directly applied in SM MIMO systems. It is because that the effect of multistream interference (MSI) makes the source separation impossible at the receiver. But if some of the information symbols in a certain MSDD block are known at the receiver as "reference symbols," because the reference symbols give the receiver some "knowledge" of the channel state and thus the source separation becomes possible, the other information symbols in that block i.e. "detection symbols" can be detected based on the reference symbols as well as the corresponding received signals of that block. If the blocks of information symbols are overlapped, the detection symbols identified for one block can also be used as the reference symbols of other blocks.

Therefore, we repartition the information symbols in an MSDD block into two parts as reference symbols and detection symbols, and define such a block as an NMLD information symbols block. We also define the NMLD information symbols blocks as being overlapped. Hence the proposed NMLD is the following recursive decision feedback process. At the very beginning, the "initial reference symbols" known to the receiver are transmitted as reference symbols to detect detection symbols in the first block; then the detected detection symbols are also used as reference symbols to detect more detection symbols in other blocks.

Note although the initial reference symbols in NMLD seem to be similar with training symbols in coherent detection methods, in fact they play different roles. In coherent detection methods, the training symbols are used to calculate the CSI and in a fast fading environment the calculated CSI will be too rough to be used. Otherwise, in NMLD the initial reference symbols are only used to initial the recursive decision feedback process, and no CSI is actually calculated.

In this paper, NMLD is considered in both slow and fast fading environments. In fast fading environments, similar to MSDD [18], the fading autocorrelation is considered for NMLD, and thus maximum Doppler frequency and noise power are included in the decision metric. Therefore, estimations of both maximum Doppler frequency and noise power are needed at the receiver for NMLD.

The rest of this paper is organized as follows. In Sect. 2, we introduce the system model. In Sect. 3, NMLD is proposed and its decision metric is derived. In Sect. 4, a fast calculation algorithm (FCA) is applied to reduce the computational complexity of NMLD. The performance analysis by computer simulation is given in Sect. 5 while the impacts of inexact estimations of maximum Doppler frequency and noise power are also discussed. And finally the conclusion is in Sect. 6.

\section{System Model}

\subsection{Channel Model}

We consider a wireless MIMO communication system with $N_{t}$ transmit antennas and $N_{r}$ receive antennas. The channels between each transmit and receive antennas are assumed to be flat Rayleigh fading and independent mutually. At time $t$, the transmitted signal from antenna $\mu$ is $s_{\mu}(t) . s_{\mu}(t)$ is selected from a $2^{m}$-PSK constellation for $m$ bit information. We assume the average transmit energy for each bit is $E_{b}$, then the transmit energy for a symbol is $m E_{b}$. The received signal $r_{v}(t)$ at antenna $v$ can be written as

$$
r_{\nu}(t)=\sum_{\mu=1}^{N_{t}} s_{\mu}(t) h_{\mu \nu}(t)+n_{\nu}(t)
$$

where $h_{\mu v}(t)$ is the zero-mean complex Gaussian path gain from the $\mu$ th transmit antenna to the $v$ th receive antenna with variance 1 , and $n_{v}(t)$ is the additive complex Gaussian noise with zero mean and one-sided power spectral density $N_{0}$ at receive antenna $v$.

All of the received signals at $N_{r}$ receive antennas can be defined as a vector $\mathbf{r}(t)=\left[r_{1}(t), \ldots, r_{N_{r}}(t)\right]$, and we can rewrite (1) into

$$
\mathbf{r}(t)=\mathbf{s}(t) \mathbf{h}(t)+\mathbf{n}(t)
$$

where $\mathbf{s}(t)=\left[s_{1}(t), \ldots, s_{N_{t}}(t)\right], \mathbf{n}(t)=\left[n_{1}(t), \ldots, n_{N_{r}}(t)\right]$ and $\mathbf{h}(t)$ is a $N_{t} \times N_{r}$ matrix with element $[\mathbf{h}(t)]_{\mu v}=h_{\mu v}(t)$.

\subsection{Differential Coding of SM MIMO}

In differential SM MIMO systems, independent signals are transmitted from all of the transmit antennas simultaneously, and the transmit signal from a certain antenna $\mu$ is differentially coded as

$$
s_{\mu}(t)=s_{\mu}(t-1) c_{\mu}(t)
$$

where $c_{\mu}(t)$ is the information symbol selected from a same $2^{m}$-PSK constellation as $s_{\mu}(t)$. We have

$$
\begin{aligned}
& s_{\mu}(t+\tau) s_{\mu}^{*}\left(t+\tau^{\prime}\right) \\
& =\left\{\begin{array}{l}
c_{\mu}(t+\tau) c_{\mu}(t+\tau-1) \ldots c_{\mu}\left(t+\tau^{\prime}+1\right) ;\left(\tau>\tau^{\prime}\right) \\
c_{\mu}^{*}\left(t+\tau^{\prime}\right) c_{\mu}^{*}\left(t+\tau^{\prime}-1\right) \ldots c_{\mu}^{*}(t+\tau+1) ;\left(\tau<\tau^{\prime}\right) \\
1 ;\left(\tau=\tau^{\prime}\right)
\end{array}\right.
\end{aligned}
$$

where the superscript ' $*$ ' denotes the complex conjugation.

Equation (3) can also be rewritten into the matrix form as

$$
\mathbf{s}(t)=\mathbf{s}(t-1) \mathbf{c}(t)
$$

where $\mathbf{c}(t)$ is a $N_{t}$ order diagonal matrix with element $[\mathbf{c}(t)]_{\mu \mu}=c_{\mu}(t)$. 


\section{NMLD for SM MIMO}

\subsection{MSDD}

With the idea of MSDD, multiple symbols of received signals are observed together as a block. We assume the observation interval is $K$, and the blocks of received signals and corresponding information symbols can be partitioned as in Figs. 1 and 2 respectively. The received signals from time $t$ to time $t+K-1$ at all the antennas can be collected in a matrix $\mathbf{R}(t)=\left[\mathbf{r}(t+K-1)^{T}, \ldots, \mathbf{r}(t)^{T}\right]^{T}$, where superscript ' $T$ ' denotes transposition, and (2) can be rewritten to

$$
\mathbf{R}(t)=\mathbf{S}(t) \mathbf{H}(t)+\mathbf{N}(t)
$$

where

$$
\begin{aligned}
& \mathbf{S}(t)=\operatorname{diag}\{\mathbf{s}(t+K-1), \ldots, \mathbf{s}(t)\} \\
& \mathbf{H}(t)=\left[\mathbf{h}(t+K-1)^{T} \ldots \mathbf{h}(t)^{T}\right]^{T} \\
& \mathbf{N}(t)=\left[\mathbf{n}(t+K-1)^{T} \ldots \mathbf{n}(t)^{T}\right]^{T} .
\end{aligned}
$$

The probability density function (pdf) of $\mathbf{R}(t)$ given $\mathbf{S}(t)$ is [19]

$$
\begin{aligned}
& p(\mathbf{R}(t) \mid \mathbf{S}(t)) \\
& =\frac{1}{\pi^{K N_{t} N_{r}} \operatorname{det} \Lambda(t)^{N_{r}}} \exp \left\{-\operatorname{tr}\left(\mathbf{R}(t)^{H} \Lambda(t)^{-1} \mathbf{R}(t)\right)\right\}
\end{aligned}
$$

where 'det,' ' $t r$ ' and the superscript ' $H$ ' denote the determinant, the trace function and the Hermitian operation (transpose conjugation) respectively, and $\Lambda(t)=$ $E\left\{\mathbf{R}(t) \mathbf{R}(t)^{H} \mid \mathbf{S}(t)\right\}$ (' $E$ ' denotes expectation) is the covariance matrix of $\mathbf{R}(t)$.

Because $\mathbf{H}(t)$ and $\mathbf{N}(t)$ are independent mutually, $\Lambda(t)$ can be simplified as

$$
\begin{aligned}
\Lambda(t) & =E\left[[\mathbf{S}(t) \mathbf{H}(t)+\mathbf{N}(t)][\mathbf{S}(t) \mathbf{H}(t)+\mathbf{N}(t)]^{H}\right] \\
& =E\left[\mathbf{S}(t) \mathbf{H}(t) \mathbf{H}(t)^{H} \mathbf{S}(t)^{H}\right]+E\left[\mathbf{N}(t) \mathbf{N}(t)^{H}\right] \\
& =\mathbf{S}(t) E\left(\mathbf{H}(t) \mathbf{H}(t)^{H}\right) \mathbf{S}(t)^{H}+\frac{N_{r} N_{0}}{2} \mathbf{I}_{K}
\end{aligned}
$$

where $\mathbf{I}_{n}$ denotes the $n$th order identity matrix.

Since we have assumed the channels between each

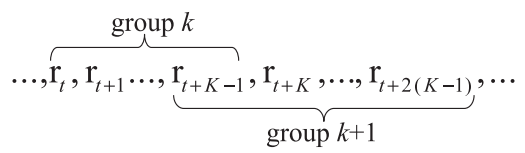

Fig. 1 Partitioning of the received signals into blocks in MSDD.

$$
\ldots, \overbrace{\mathrm{C}_{t+1}, \mathrm{c}_{t+2}, \ldots, \mathrm{c}_{t+K-1}}^{\text {group } k}, \underbrace{\mathrm{c}_{t+K}, \mathrm{c}_{t+K+1} \ldots, \mathrm{c}_{t+2(K-1)}}_{\text {group } k+1}, \cdots
$$

Fig. 2 Partitioning of the corresponding information symbols into blocks in MSDD. transmit and receive antennas are independent mutually, we have

$$
E\left\{h_{\mu \nu}(t) h_{\mu^{\prime} v^{\prime}}^{*}(t)\right\}=0 \quad\left(\mu \neq \mu^{\prime} \quad \text { and } / \text { or } \quad v \neq v^{\prime}\right) .
$$

And the fading autocorrelation can be modeled as [20]

$$
\rho\left(t-t^{\prime}\right)=E\left\{h_{\mu v}(t) h_{\mu v}^{*}\left(t^{\prime}\right)\right\}=J_{0}\left(2 \pi f_{D} T_{s}\left(t-t^{\prime}\right)\right)
$$

where $f_{D}$ is the maximum Doppler frequency, $T_{s}$ is the sample period, and $J_{0}(\cdot)$ is the zeroth order Bessel function of the first kind. Therefore

$$
E\left[\mathbf{H}(t) \mathbf{H}(t)^{H}\right]=N_{r} \boldsymbol{\Gamma} \otimes \mathbf{I}_{N_{t}}
$$

where $\otimes$ denotes the Kronecker product and $\boldsymbol{\Gamma}$ is a $K \times K$ matrix of the fading autocorrelation as

$$
\boldsymbol{\Gamma}=\left[\begin{array}{cccc}
\rho(0) & \rho(1) & \cdots & \rho(K-1) \\
\rho(-1) & \rho(0) & \cdots & \rho(K-2) \\
\vdots & \ddots & \ddots & \vdots \\
\rho(1-K) & \cdots & \cdots & \rho(0)
\end{array}\right]
$$

We have

$$
\Lambda(t)=N_{r} \mathbf{S}(t)\left(\boldsymbol{\Gamma} \otimes \mathbf{I}_{N_{t}}\right) \mathbf{S}(t)^{H}+\frac{N_{r} N_{0}}{2} \mathbf{I}_{K} .
$$

We define $\lambda(t)_{i j}$ as the $i$,jth element of $\Lambda(t)$ thus

$$
\begin{aligned}
& \lambda(t)_{i j} \\
& =N_{r}\left(\sum_{\mu=1}^{N_{t}} s_{\mu}(t+K-i) s_{\mu}^{*}(t+K-j) \rho(i-j)+\frac{N_{0}}{2} \delta_{i j}\right)
\end{aligned}
$$

where $\delta_{i j}=\left\{\begin{array}{l}1 ; i=j \\ 0 ; i \neq j\end{array}\right.$

Since the natural logarithm is a monotonically increasing function of its argument, maximizing $p(\mathbf{R}(t) \mid \mathbf{S}(t))$ is equivalent to maximizing $\ln p(\mathbf{R}(t) \mid \mathbf{S}(t))$. And because $s_{\mu}(t)$ is selected from a $2^{m}$-PSK constellation, det $\Lambda(t)$ is independent with $\mathbf{S}(t)$. By taking logarithm of (7) and ignoring the terms which are independent of $\mathbf{S}(t)$, we can get the decision metric of MSDD for SM MIMO systems as

$$
\eta=-\operatorname{tr}\left(\mathbf{R}(t)^{H} \Lambda(t)^{-1} \mathbf{R}(t)\right)
$$

\section{$3.2 \quad$ NMLD}

We define $C_{\mu}^{t}=\left\{c_{\mu}(t+1), \ldots, c_{\mu}(t+K-1)\right\}$ as the set of a block of information symbols from transmit antenna $\mu$. From (4), (14) and (15), we can observe that the value of $C_{\mu}^{t}$ has the same effect on the decision metric as the value of $C_{\mu^{\prime}}^{t}$. That means the information symbols from different transmit antennas can not be separated, therefore by only maximizing (15) the information symbols can not be decided. We can also observe that the effect on the decision metric of a certain information symbol $c_{\mu}(\tau) \in C_{\mu}^{t}$ always depends on 


$$
\ldots, \overbrace{\mathrm{r}_{t}, \ldots, \underbrace{\mathrm{r}_{t+N}, \ldots, \mathrm{r}_{t+L}, \ldots, \mathrm{r}_{t+K-1}}_{\text {group } k+1}, \ldots, \mathrm{r}_{t+K+N-1}}^{\text {group } k}, \ldots
$$

Fig. 3 Partitioning of the received signals into blocks in proposed NMLD.

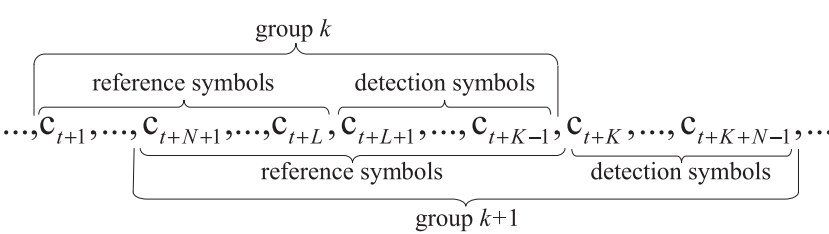

Fig. 4 Partitioning of the corresponding information symbols into blocks in proposed NMLD.

the other information symbols in $C_{\mu}^{t}$. If some information symbols of each $C_{\mu}^{t}$, which have different values if transmitted from different antennas, are known to the receiver, the receiver can use those elements as reference symbols and the decision of the other information symbols becomes possible.

To make (15) available for SM MIMO, we repartition received signals and the corresponding information symbols into blocks with interval length $K$ and $K-1$ as shown in Figs. 3 and 4 respectively. The $K-1$ information symbols in a certain block are divided into reference symbols with length $L$ and detection symbols with length $N$, and the blocks of information symbols are overlapped. Reference symbols are assumed to be known at the receiver and used to help to decide the detection symbols; when the detection symbols are detected, they will also be used as a part of reference symbols for other blocks to help to decide the detection symbols. To initialize the above decision feedback process, we assume an initial reference symbol series known to the receiver with length $L$ is transmitted at the very beginning from every transmit antennas as the reference symbols for the first block. And the initial reference symbol series from different antennas are different mutually.

With the partitioning above, $\Lambda(t)$ can be reformed into $\Lambda^{\prime}(t)$ and (4) can be rewritten into

$$
\begin{gathered}
s_{\mu}(t+\tau) s_{\mu}^{*}\left(t+\tau^{\prime}\right) \\
=\left\{\begin{array}{c}
c_{\mu}(t+\tau) \ldots c_{\mu}\left(t+\tau^{\prime}+1\right) ;\left(\tau>\tau^{\prime} \geq L\right) \\
c_{\mu}(t+\tau) \ldots c_{\mu}(t+L+1) \hat{c}_{\mu}(t+L) \ldots \hat{c}_{\mu}\left(t+\tau^{\prime}+1\right) ; \\
\left(\tau>L>\tau^{\prime}\right) \\
\hat{c}_{\mu}(t+\tau) \ldots \hat{c}_{\mu}\left(t+\tau^{\prime}+1\right) ;\left(L \geq \tau>\tau^{\prime}\right) \\
c_{\mu}^{*}\left(t+\tau^{\prime}\right) \ldots c_{\mu}^{*}(t+\tau+1) ;\left(L \leq \tau<\tau^{\prime}\right) \\
c_{\mu}^{*}\left(t+\tau^{\prime}\right) \ldots c_{\mu}^{*}(t+L+1) \hat{c}_{\mu}^{*}(t+L) \ldots \hat{c}_{\mu}^{*}(t+\tau+1) ; \\
\left(\tau<L<\tau^{\prime}\right) \\
\hat{c}_{\mu}^{*}\left(t+\tau^{\prime}\right) \ldots \hat{c}_{\mu}^{*}(t+\tau+1) ;\left(\tau<\tau^{\prime} \leq L\right) \\
1 ;\left(\tau=\tau^{\prime}\right)
\end{array}\right.
\end{gathered}
$$

where $\hat{c}_{\mu}(t)$ is the reference symbol and $c_{\mu}(t)$ is the detection symbol. By putting (16) into (14), the element of $\Lambda^{\prime}(t)$ can be got (which is omitted here for simplicity). Then finally we get the decision metric of NMLD as

$$
\eta=-\operatorname{tr}\left(\mathbf{R}(t)^{H} \Lambda^{\prime}(t)^{-1} \mathbf{R}(t)\right) .
$$

\section{FCA for NMLD}

We notice that the computational complexity of (17) mainly depends on calculating the inverse matrix of $\Lambda^{\prime}(t)$. Since there are $N$ detection symbols in a block and each of them has $2^{m N_{t}}$ possible values, $\Lambda^{\prime}(t)$ has $2^{m N_{t} N}$ possible values. Therefore in NMLD, we have to compute the inverse matrix of $2^{m N_{t} N} K \times K$ matrices. To reduce the complexity of calculating $\Lambda^{\prime-1}(t)$, FCA proposed in [18] is applied.

In FCA, $\mathbf{A}_{l}$ is assumed to be an $l \times l$ reversible matrix and partitioned into four submatrices as

$$
\begin{aligned}
\mathbf{A}_{l} & =\left[\begin{array}{ccc|c}
a_{11} & \cdots & a_{1(l-1)} & a_{1 l} \\
\vdots & \cdots & \cdot & \vdots \\
a_{(l-1) 1} & \cdot & a_{(l-1)(l-1)} & a_{(l-1) l} \\
\hline a_{l 1} & \cdots & a_{l(l-1)} & a_{l l}
\end{array}\right] \\
& =\left[\begin{array}{cc}
\mathbf{A}_{l-1} & \mathbf{A}_{1 l} \\
\mathbf{A}_{l 1} & a_{l l}
\end{array}\right]
\end{aligned}
$$

Assuming $\mathbf{B}_{l}=\mathbf{A}_{l}^{-1}$ as the inverse matrix of $\mathbf{A}_{l}$, accordingly $\mathbf{B}_{l}$ is partitioned as

$$
\mathbf{B}_{l}=\left[\begin{array}{cc}
\mathbf{B}_{(l-1)(l-1)} & \mathbf{B}_{1 l} \\
\mathbf{B}_{l 1} & b_{l l}
\end{array}\right]
$$

If $\mathbf{A}_{l}=\mathbf{A}_{l}^{H}$, the submatrices of $\mathbf{B}_{l}$ can be calculated as

$$
\begin{aligned}
& b_{l l}=\left(a_{l l}-\mathbf{A}_{l 1} \mathbf{A}_{l-1}^{-1} \mathbf{A}_{1 l}\right)^{-1} \\
& \mathbf{B}_{1 l}=-\mathbf{A}_{l-1}^{-1} \mathbf{A}_{1 l} b_{l l}=\mathbf{B}_{l 1}^{H} \\
& \mathbf{B}_{(l-1)(l-1)}=\mathbf{A}_{l-1}^{-1}-\mathbf{B}_{1 l} \mathbf{A}_{l 1} \mathbf{A}_{l-1}^{-1}
\end{aligned}
$$

That means, with FCA the process of calculating the inverse matrix of a $l$ th order matrix $\mathbf{A}_{l}$ with property $\mathbf{A}_{l}=\mathbf{A}_{l}^{H}$ can be converted to calculating the inverse matrix of its $(l-1)$ th order submatrix $\mathbf{A}_{l-1}$ instead; by applying FCA recursively, it can also be converted to calculating the inverse matrix of any of its lower order submatrix $\mathbf{A}_{l-i},(i<l)$. Note just only apply FCA can not actually reduce the computational complexity.

In our case of NMLD, it can be observed from (16) that if both of the $\tau$ and $\tau^{\prime}$ are not more than $L, s_{\mu}(t+\tau) s_{\mu}^{*}\left(t+\tau^{\prime}\right)$ has a certain value. That means $\lambda^{\prime}(t)_{i, j}$, which is the $i$, jth element of $\Lambda^{\prime}(t)$, has a certain value if both $i$ and $j$ are not more than $L+1$. We define $\Lambda_{l}^{\prime}(t)(l \leq K)$ as

$$
\Lambda_{l}^{\prime}(t)=\left[\begin{array}{ccc}
\lambda^{\prime}(t)_{11} & \cdots & \lambda^{\prime}(t)_{1 l} \\
\vdots & \cdots & \vdots \\
\lambda^{\prime}(t)_{l 1} & \vdots & \lambda^{\prime}(t)_{l l}
\end{array}\right]
$$

thus $\Lambda^{\prime}(t)=\Lambda_{K}^{\prime}(t)$, and $\Lambda_{l}^{\prime}(t)$ is a certain matrix if $l \leq L+1$. 
It is obviously $\Lambda^{\prime}(t)=\Lambda^{\prime H}(t)$, with FCA the calculation of $\Lambda^{\prime-1}(t)$ can be converted to the calculation of $\Lambda_{L+1}^{-1}(t)$. Since $\Lambda_{L+1}^{\prime}(t)$ is a certain matrix, to get $2^{m N_{t} N}$ possible values of $\Lambda^{\prime-1}(t)$, instead of calculating the inverse matrix of $2^{m N_{t} N}$ $K \times K$ matrices, we can calculate the inverse matrix of an $(L+1) \times(L+1)$ matrix and applying FCA. And the computational complexity of such a process mainly depends on computing the inverse matrix of an $(L+1) \times(L+1)$ matrix and using it to calculate all the possible values. Therefore the computational complexity is highly reduced.

\section{Simulation Results}

In our simulation, a time-varying flat Rayleigh fading MIMO channel model is used. The number of transmit antennas $N_{t}$ and receive antennas $N_{r}$ are both set to 2 if not stated otherwise. The transmit antennas are assumed to be independent with each other as well as receive antennas. Transmitted signals and information symbols are selected from a QPSK (quadrature phase shift keying) constellation. At the very beginning, initial reference symbol series, which are known to the receiver, with length $L$ are transmitted from every transmit antennas, and the initial reference symbol series from different antennas are different mutually. And the initial reference symbols are retransmitted after detecting every 102 detection symbols. The normalized maximum Doppler frequency $f_{D} T_{s}$ is set to 0.001 for slow fading channels and to 0.01 for fast fading channels. Unlike in [8], [9] and [10], estimations of $f_{D}$ and $N_{0}$ are needed at the receiver in the proposed NMLD. For the moment we assume $f_{D}$ and $N_{0}$ are exactly estimated at the receiver, i.e. $\hat{f}_{D}=f_{D}$, $\hat{N}_{0}=N_{0}$, where $\hat{f}_{D}$ and $\hat{N}_{0}$ are the estimated $f_{D}$ and $N_{0}$ at the receiver respectively.

Figures 5 and 6 show the BER performances of NMLD with differential reference symbol length $L$ and detection symbol length 1 for slow and fast fading channel respectively. Performance of coherent MLD with perfect CSI for

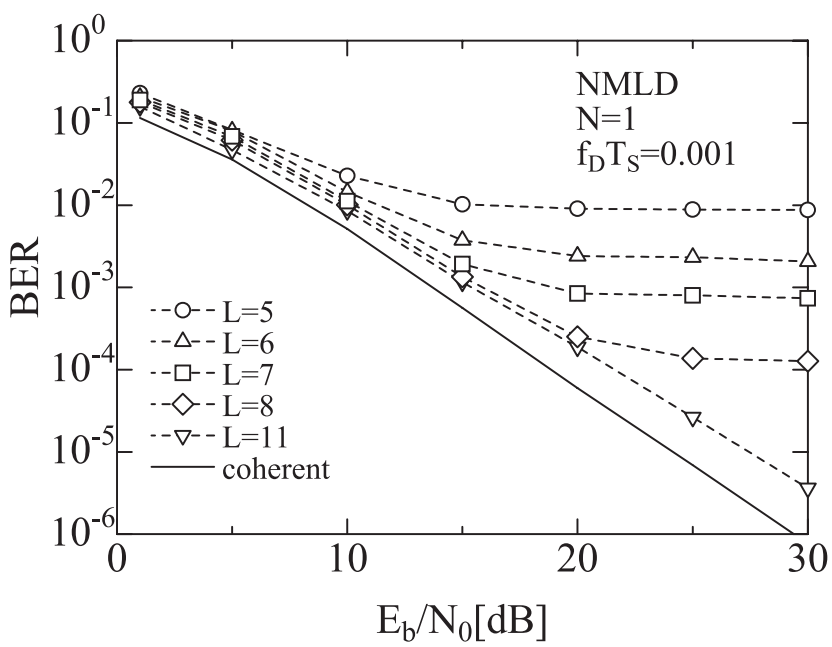

Fig. 5 The average BER performance of NMLD in slow fading channels with different length of reference symbol interval. differential SM MIMO is also included for comparison. In these figures, we can observe that in slow fading channel with a small reference symbol length $(L \leq 8)$, increase in the signal-to-noise rate can hardly lead to an improvement of BER when $E_{b} / N_{0}$ is big. It is because that when the reference symbol length is small, the receiver can not get enough "knowledge" of the channel, and the effect of MSI cannot be totally canceled. We can also observe that with the increase of reference symbol length and when the reference symbol length is big enough $(L=11)$ the BER floor is no longer existed. That is because the more reference symbols are used, the receiver will have the better "knowledge" of channel and the effect of MSI accordingly becomes less; and when the reference symbol length is big enough, the effect of MSI can be almost totally canceled.

The BER performances of NMLD with differential detection symbol length $N$ are shown in Figs. 7 and 8 for slow and fast fading respectively. We can observe that increasing the length of detection symbols can also lead to some

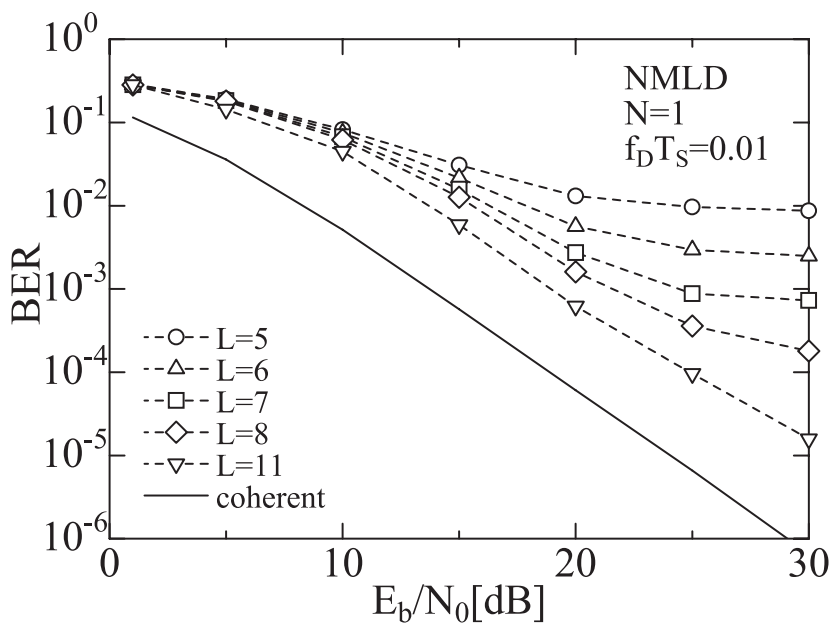

Fig. 6 The average BER performance of NMLD in fast fading channels with different length of reference symbol interval.

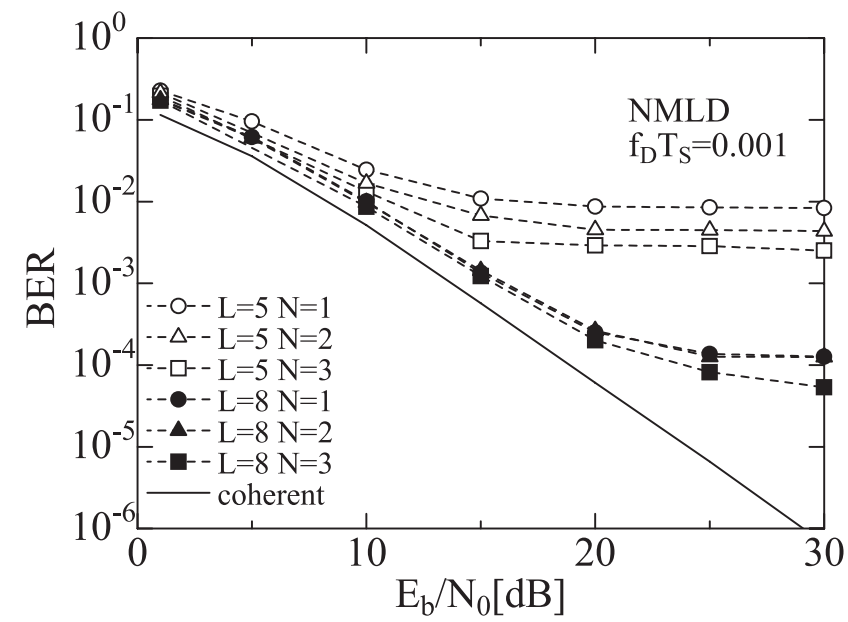

Fig. 7 The average BER performance of NMLD in slow fading channels with different length of reference and detection symbol interval. 


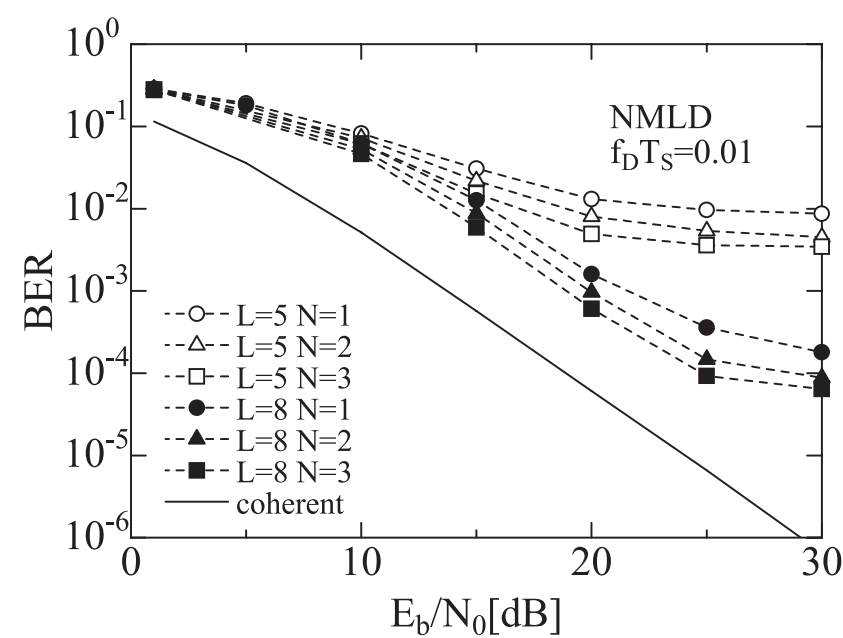

Fig. 8 The average BER performance of NMLD in fast fading channels with different length of reference and detection symbol interval.

performance gain, but the gain is much less than that by increasing the reference symbol length. That is because extending the reference symbol length leads to a less MSI effect while the meaning of extending the detection symbol length is to compensate for the performance loss between differential and coherent detection, and the effect of such a performance loss is much less than the MSI effect. And since the extension of detection symbol length will lead to a much bigger computational complexity increase, increasing the reference symbols tends to be chosen as the way to improvement the performance of NMLD.

The performances of DV-BLAST, DSM, PADE and NMLD are compared next. As discussed previously, in NMLD, we assumed that an initial reference symbol series known to the receiver is transmitted. For DSM, a similar training sequence known to the receiver is also assumed to be transmitted. Such assumptions are necessary for NMLD and DSM to initialize the detection process. But to meet the assumptions, the data rate of the whole system is slightly lowered and the system complexity is increased. In contrast, for DV-BLAST and PADE, such kind of assumption is not need to be made.

The BER performances of DV-BLAST, DSM, PADE and NMLD in slow fading channels are compared in Fig. 9. Here a BPSK (binary phase shift keying) constellation is used for DV-BLAST, the observation window size of DFDD is set to 2 for DSM and performance improvement scheme in [16] is applied for PADE. When $f_{D} T_{s}=0$, it can be observed that NMLD has better performance than DV-BLAST, DSM and PADE. When $f_{D} T_{s}$ increases to 0.001 , though NMLD has only a little performance deterioration, the performance deterioration of DSM is very large.

In Fig. 10, the BER performances of NMLD and PADE in fast fading channels are compared. With $N_{r}=2$, it can be observed that, when $E_{b} / N_{0}$ is small, NMLD has only a little performance improvement compared with PADE; when $E_{b} / N_{0}$ is big, PADE suffers from BER floor while NMLD does not. With $N_{r}=4$, even in small $E_{b} / N_{0}$ condition,

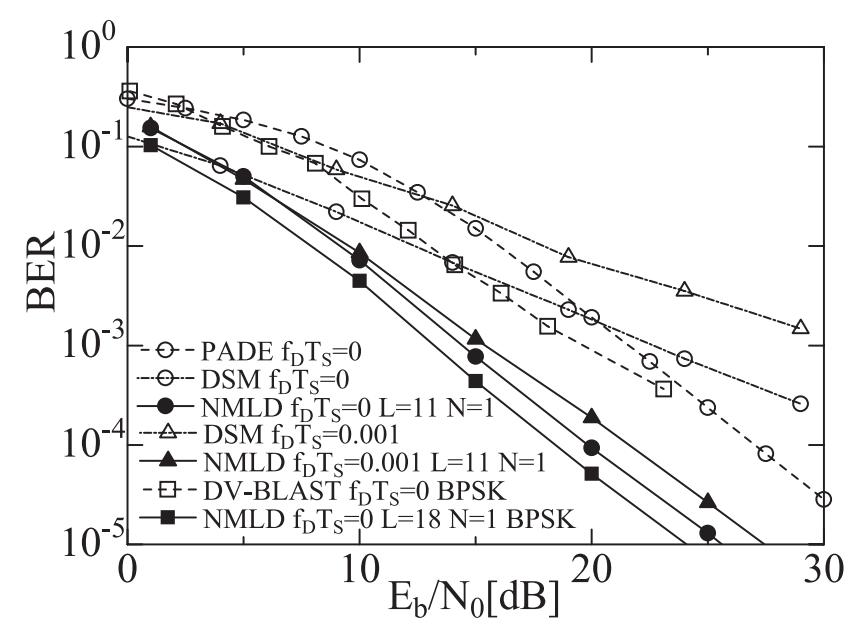

Fig. 9 The average BER performance of DV-BLAST, DSM, PADE and NMLD in slow fading channels.

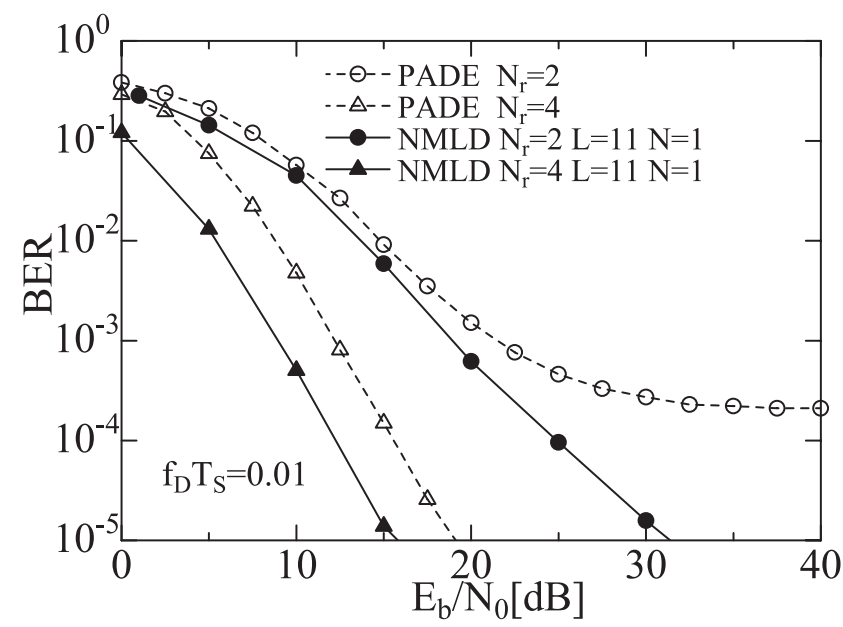

Fig. 10 The average BER performance of NMLD and PADE in fast fading channels with different number of receive antennas.

Table 1 Computational complexity of DV-BLAST, DSM, PADE and NMLD.

\begin{tabular}{c||c|c|c|c|}
\hline & DV-BLAST & DSM & PADE & NMLD \\
\hline$N_{A}$ & 2 & 15 & 3 & 1240 \\
\hline$N_{B}$ & 4 & 8 & 256 & 8 \\
\hline$N_{C}$ & 0 & 95 & 0 & 0 \\
\hline$N_{D}$ & 8 & 215 & 768 & 9920 \\
\hline
\end{tabular}

NMLD has much better BER performance than PADE.

In Table 1, the computational complexities of DVBLAST, DSM, PADE and NMLD are compared. Here we set $N_{t}=2, N_{r}=2$ and QPSK constellation is used. Furthermore, we set $L=11, N=1$ for NMLD, and the observation window size of DFDD is set to 2 for DSM. In Table $1, N_{A}$ is the number of multiplication operations performed in a decision metric (for DV-BLAST, DSM and NMLD) or branch metric (for PADE); $N_{B}$ is the average number of metrics calculated to estimate one information symbol; $N_{C}$ is the average number of multiplication operations performed to calculate the filter parameters for one information symbol (only 


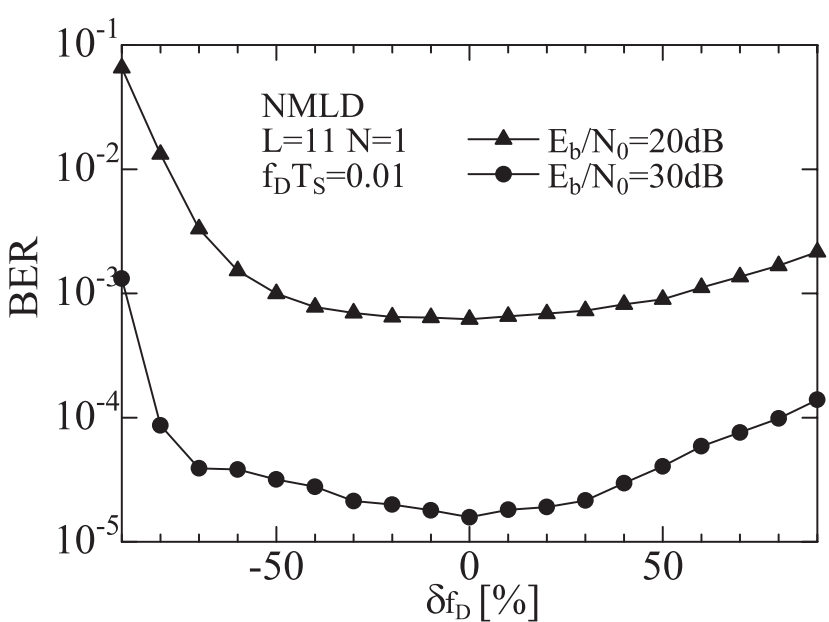

Fig. 11 The average BER performance of NMLD in fast fading channels with inexact estimation of $f_{D}$.

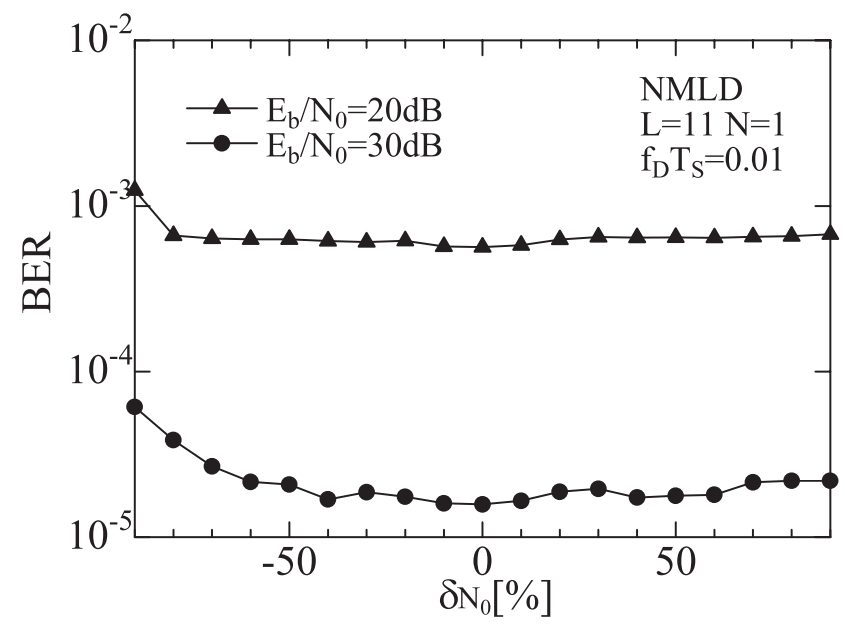

Fig. 12 The average BER performance of NMLD in fast fading channels with inexact estimation of $N_{0}$.

for DSM); and $N_{D}=N_{A} N_{B}+N_{C}$ is the average number of multiplication operations performed to estimate one information symbol.

It can be observed from Table 1 that NMLD has much larger computational complexity than other methods. That means the superiority of NMLD on BER performance depends on the additional cost of a large computational complexity.

So far, we have assumed exact estimation of $f_{D}$ and $N_{0}$ at the receiver. To estimate $f_{D}$ and $N_{0}$ at the receiver, there are some methods, such as [21] and [22], can be used separately. But estimation errors are inevitable in practical systems. Since the estimations of $f_{D}$ and $N_{0}$ are very essential for our method, the impacts of estimation errors in $f_{D}$ and $N_{0}$ on the BER performance are also investigated. Here we set the relative errors of $f_{D}$ and $N_{0}$ as $\delta_{f_{D}}=\left(\hat{f}_{D}-f_{D}\right) / f_{D}$ and $\delta_{N_{0}}=\left(\hat{N}_{0}-N_{0}\right) / N_{0}$ respectively.

Figure 11 shows the BER performances of NMLD in fast fading channels with inexact estimation of $f_{D}$ at the re- ceiver. It can be observed that the estimation errors lead to some performance deteriorations. When $\left|\delta_{f_{D}}\right| \leq 50 \%$, the deterioration rate is low and the BER performance is acceptable. That means an estimation method of $f_{D}$ with $\left|\delta_{f_{D}}\right| \leq 50 \%$ can meet our needs for NMLD.

The BER performances of NMLD in fast fading channels with inexact estimation of $N_{0}$ at the receiver are shown in Fig. 12. It is observed that the BER performance is hardly affected by the estimation errors in $N_{0}$ when $\left|\delta_{N_{0}}\right| \leq 70 \%$. And even when $\delta_{N_{0}}=-90 \%$, the BER performance is still not unacceptable. That means NMLD is quite insensitive to the estimation errors in $N_{0}$. Therefore, even a rough estimation method of $N_{0}$ can meet our needs for NMLD.

\section{Conclusion}

In this paper, a novel NMLD method for SM MIMO systems, which can avoid channel estimation, has been proposed. After repartitioning the observation intervals of MSDD and following a decision feedback process, the decision metric of noncoherent MLD is derived by reforming the decision metric of MSDD for SM MIMO systems. FCA is applied to reduce the computational complexity of NMLD. The feasibility of the proposed NMLD has been demonstrated by computer simulations in both slow and fast fading environments. Simulation results show that the BER performance of proposed NMLD is approaching that of the conventional coherent MLD with the extension of reference symbols interval and has only a little performance loss with a large $L$. It is also shown that extending the detection symbols interval can also lead to some performance gain. Increasing the reference symbol length is more competitive between the two ways, since it leads to a much better gain and has lower computational complexity comparing with increasing the detection symbol length. Simulation results also prove that with exact estimation of $f_{D}$ and $N_{0}$, the performance of NMLD overcomes that of the DV-BLAST, DSM and PADE. Finally the impacts of estimation errors in $f_{D}$ and $N_{0}$ have also been investigated.

\section{References}

[1] G.J. Foschini and M.J. Gans, "On limits of wireless communication in a fading environment when using multiple antennas," Wirel. Pers. Commun., vol.6, no.3, pp.311-335, March 1998.

[2] S.M. Alamouti, "A simple transmit diversity technique for wireless communications," IEEE J. Sel. Areas Commun., vol.16, pp.14511458, Oct. 1998.

[3] V. Tarokh, H. Jafarkhani, and A.R. Calderbank, "Space-time block codes from orthogonal designs," IEEE Trans. Inf. Theory, vol.45, pp.1456-1467, July 1999.

[4] G.J. Foschini, "Layered space-time architecture for wireless communication in a fading environment when using multi-element antennas," Bell Labs Tech. J., pp.41-59, Autumn 1996.

[5] H. Bolcskei, D. Gesbert, and A.J. Paulraj, "On the capacity of OFDM-based spatial multiplexing systems," IEEE Trans. Commun., vol.50, pp.225-234, Feb. 2002.

[6] B.A. Bjerke and J.G. Proakis, "Multiple-antenna diversity techniques for transmission over fading channels," Proc. IEEE WCNC'99, pp.1038-1042, Sept. 1999. 
[7] G.D. Golden, C.J. Foschini, R.A. Valenzuela, and P.W. Wolniansky, "Detection algorithm and initial laboratory results using VBLAST space-time communication architecture," Electron. Lett., vol.35, pp.14-16, 7 Jan. 1999.

[8] D. Sun, Y. Tang, S. Shao, and S. Li, "Differential space-time modulation for modified V-BLAST system," Proc. FGCN 2007, vol.2, pp.223-227, Dec. 2007.

[9] S.K. Cheung and R. Schober, "Differential spatial multiplexing," IEEE Trans. Commun., vol.5, pp.2127-2135, Aug. 2006.

[10] H. Kubo, M. Higashinaka, A. Okazaki, B. Penther, and K. Murakami, "MIMO communication systems employing per transmit antenna differential mapping (PADM)," Proc. VTC 2004-Spring, vol.2, pp.613-617, May 2004.

[11] S. Shao, Y. Tang, J. Liang, X. Li, and S Li, "A modified V-BLAST system for performance improvement through introducing different delay offsets to each spatially multiplexed data streams," Proc. IEEE WCNC 2007, pp.1062-1067, March 2007.

[12] H.-j. Su and E. Geraniotis, "Maximum signal-to-noise ratio array processing for space-time coded systems," IEEE Trans. Commun., vol.50, pp.1419-1422, Sept. 2002.

[13] R. Schober, W.H. Gerstacker, and J.B. Huber, "Decision-feedback differential detection of MDPSK for flat Rayleigh fading channels," IEEE Trans. Commun., vol.47, pp.1025-1035, July 1999.

[14] G. Forney, Jr., "Maximum-likelihood sequence estimation of digital sequences in the presence of intersymbol interference," IEEE Trans. Inf. Theory, vol.IT-18, no.3, pp.363-378, May 1972.

[15] R. Raheli, A. Polydoros, and C.-K. Tzou, "Per-survivor processing: A general approach to MLSE in uncertain environments," IEEE Trans. Commun., vol.43, pp.354-364, Feb./March/April 1995

[16] H. Kubo, M. Higashinaka, A. Okazaki, B. Penther, and K. Murakami, "Performance improvement for MIMO communication systems employing per transmit antenna differential encoding (PADE)," Proc. IEEE PIMRC 2005, vol.1, pp.42-46, Sept. 2005.

[17] D. Divsalar and M.K. Simon, "Multiple-symbol differential detection of MPSK," IEEE Trans. Commun., vol.38, pp.300-308, March 1990.

[18] S. Handa, Y. Okano, M. Liu, F. Sasamori, and S. Oshita, "Fast calculation algorithm and error performance of multiple-symbol differential detection over fading channels," IEICE Trans. Commun., vol.E86-B, no.3, pp.1050-1056, March 2003.

[19] R. Schober and L.H.-J. Lampe, "Noncoherent receivers for differential space-time modulation,” IEEE Trans. Commun., vol.50, pp.768777, May 2002.

[20] W.C. Jakes, ed., Microwave Mobile Communications, Wiley, New York, 1974

[21] Y.R. Zheng and C. Xiao, "Mobile speed estimation for broadband wireless communications over Rician fading channels," IEEE Trans. Wireless Commun., vol.8, no.1, pp.1-5, Jan. 2009.

[22] C.-S. Tsang, G.L. Lui, and K.T. Woo, "Algorithm and performance of a noncoherent SNR estimator," Proc. IEEE MILCOM'95, vol.1, pp.155-159, Nov. 1995.

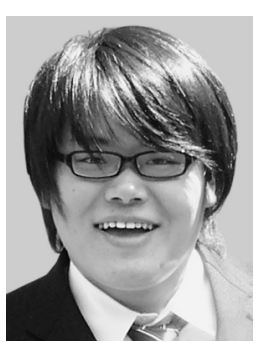

Katsunobu Yoshii received the B.E. and M.E. degrees in Electrical and Electronic Engineering from Shinshu University in 2006 and 2008 respectively. He engaged in research of MIMO techniques at the university. He has been with Panasonic Electric Works Corporation from 2008.

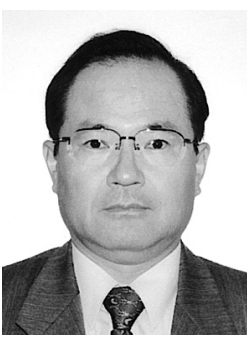

Shiro Handa received the B.E. and M.E. degrees from Shinshu University in 1978 and 1980 respectively, and the Dr.Eng. degree from Kobe University in 1988. From 1982 to 1988, he was a Research Associate at Kobe University. From 1988 to 1994 , he was with Nagano National College of Technology. He has been with the Department of Electrical and Electronic Engineering, Shinshu University, since 1994 as an Associate Professor and since 2005 as a Professor In 1996, he was at the University of California Davis, as a visiting researcher. His research interests include satellite and mobile communication systems, modulation and coding, and data compression. He is a member of IEEE and SITA.

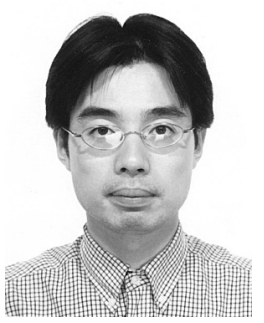

Fumihito Sasamori received the B.E., M.E and Dr.Eng. degrees from Waseda University, Tokyo in 1994, 1996 and 2000, respectively. Since 2000 he has been with the Department of Electrical and Electronic Engineering, Shinshu University, first as a Research Associate and since 2006 as an Associate Professor. His current research interests include digital mobile communication systems. He received the IEICE Young Engineer Award in 2000. He is a member of IEEE.

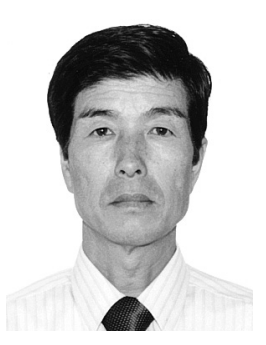

Shinjiro Oshita received the B.E. degree from Osaka Electro Communication University in 1967, the M.E. and Dr.Eng. degrees from Osaka University, Japan in 1970 and 1973, respectively. Since 1973, he has been with Shinshu University, Nagano, Japan, where he is currently a Professor in the Department of Electrical and Electronic Engineering. His research interests include intelligent CAI and communications theory. He is a member of IEEE and Japanese Society for Information and System in

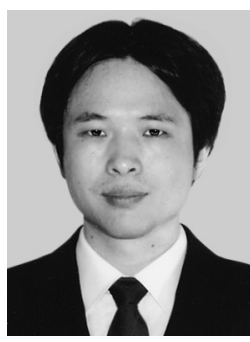

Ziyan Jia received the B.E. degree from Nanjing University of Posts and Telecommunications, China in 2004, and the M.E. degree from Shinshu University, Japan in 2007. He is currently pursuing the Dr.Eng. degree in the Department of Electrical and Electronic Engineering, Shinshu University. His current research interests include coding and modulation for mobile communications.
Education

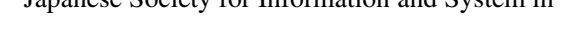

